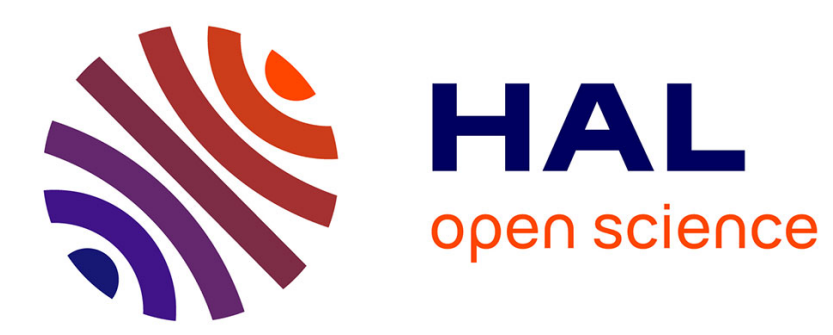

\title{
Aspects physiologiques de la pourriture des tiges du maïs
}

\author{
Yves Barriere
}

\section{To cite this version:}

Yves Barriere. Aspects physiologiques de la pourriture des tiges du maïs. Agronomie, 1985, 5 (4), pp.361-368. hal-00884770

\section{HAL Id: hal-00884770 \\ https://hal.science/hal-00884770}

Submitted on 1 Jan 1985

HAL is a multi-disciplinary open access archive for the deposit and dissemination of scientific research documents, whether they are published or not. The documents may come from teaching and research institutions in France or abroad, or from public or private research centers.
L'archive ouverte pluridisciplinaire HAL, est destinée au dépôt et à la diffusion de documents scientifiques de niveau recherche, publiés ou non, émanant des établissements d'enseignement et de recherche français ou étrangers, des laboratoires publics ou privés. 


\title{
Aspects physiologiques de la pourriture des tiges du maiis
}

\author{
Yves BARRIERE \\ INRA, Station d'Amélioration des Plantes Fourragères, F 86600 Lusignan
}

\begin{abstract}
Des notations réalisées sur 8 hybrides de sensibilité connue à la fusariose du maïs montrent l'importance des phénomènes liés aux équilibres source-puits. Les génotypes résistants tendent à avoir un rythme d'apparition des feuilles adultes au stade jeune plus élevé. lls présentent un indice de surface foliaire (LAl) plus élevé en fïn de saison, tandis que le LAI à floraison ne semble pas distinguer les génotypes résistants des génotypes sensibles. La sénescence des tiges est précoce et intense chez les génotypes sensibles, mais l'évolution de certains génotypes résistants est très proche de celle des sensibles pour ce caractère. Les génotypes sensibles semblent avoir une teneur en matière sèche des tiges plus faible 1 mois après la floraison, correspondant peut-être à un vidage des tiges plus précoce et plus important, et une teneur plus élevée en fin de saison, correspondant à une sénescence plus élevée des tissus. Les rapports épi/plante entière sont plus élevés en fin de saison pour les génotypes sensibles. Toutefois, il ne semble pas exister une physiologie de plante sensible et une physiologie de plante résistante. Ce sont les équilibres nutritionnels qui s'instaurent au cours du cycle, en conséquence de l'ensemble des relations physiologiques de la plante entière et des facteurs du milieu (froid, semi-parasites, éventuellement parasites), qui conduisent à la sensibilité ou à la résistance.
\end{abstract}

Mots clés additionnels : Maïs-fourrage, fusariose.

\begin{abstract}
Physiological aspects of stalk rot in maize.
The importance of source-sink relationships as factors in resistance of maize to Fusarium stalk rot has been shown by physiological measurements on 8 genotypes of known susceptibility to the disease. Resistant genotypes probably had a higher adult-leaf appearance rate during early development, but no differences were observed for present leaf appearance rate (table 2). Resistant genotypes had a higher leaf-area index (LAI) 1 month before grain harvest time, but LAI at mid-silking stage was the same for susceptible and resistant genotypes (table 3, fig. 1). Basal internode senescence began early, at a high rate, for susceptible genotypes, but some resistant ones did not greatly differ from them (table 4, fig. 2). The susceptible genotypes studied had a lower content of stalk dry matter one month after midsilking, perhaps because of earlier migration of carbohydrates from stalk to ear, and a higher one at the end of the season, because of greater senescence of the stalk tissues (fig. 3). The whole ear/whole plant ratio was higher for susceptible genotypes during the last period of growth (table 5, fig. 4). It was concluded that there is probably not one physiological pattern for susceptible genotypes and another for resistant genotypes, but that the nutritional balance that occurs throughout the growth of the whole plant as a result of physiological processes and of external factors such as chilling injury or susceptibility to weakly parasitic fungi, leads to susceptibility or resistance.
\end{abstract}

Additional key words : Forage-maize, Fusarium stalk rot.

\section{INTRODUCTION}

La pourriture des tiges du maïs est maintenant envisagée comme la conséquence de désordres physiologiques autant que d'agressions parasitaires. Ces différents désordres sont, en particulier, liés à la sénescence des plantes et aux équilibres source-puits qui s'établissent pendant la phase de remplissage du grain. Par des notations de caractères liés aux évolutions physiologiques des plantes sur des génotypes de sensibilité connue à la " fusariose ", nous avons recherché l'importance respective de différents facteurs susceptibles d'expliquer le symptôme tiges (et racines) pourries.

\section{MATÉRIELS ET MÉTHODES}

Huit hybrides de précocité comprise entre des indices FAO 180 et FAO 280 ("Primeur », « Leader», "Brulouis", "Brussol », "Bruex", « Dea ", "F16 × F19 », "Monarque ») ont été étudiés à la 
densité de 85000 plantes/ha à Lusignan (Vienne) en 1982 et 1983, à Estrées-Mons (Somme) en 1983 avec un protocole simplifié, et à St-Martin-de-Hinx (Landes) en 1983 pour une notation de pourriture des tiges, avec une disposition en blocs à 3 répétitions. Les productions de matière sèche des différents organes (tiges, feuilles + gaines, épis) ainsi que leurs teneurs en matière sèche ont été estimées par un prélèvement de 10 (ou 6) plantes par répétition 1 et 4 semaines après la floraison de chacun des hybrides à Lusignan en 1982, 1 semaine, 1,2 et 3 mois après la floraison à Lusignan en 1983, 3 semaines et 3 mois après la floraison à Estrées-Mons en 1983. Une étude du rythme d'apparition des feuilles a été réalisée à Lusignan en 1982 entre les 28 mai et 29 juin, à partir du stade 2 feuilles adultes (feuille cotylédonaire incluse) jusqu'au stade 13-14 feuilles visibles qui correspond au nombre de feuilles total des génotypes précoces. Six notations ont été réalisées à des dates qui correspondaient à des sommes de températures en base $6{ }^{\circ} \mathrm{C}$ après le semis de $195,287,387,436,509,584$ degrésjour. Des mesures de nombre de feuilles et de surface foliaire de la plante entière (longueur $\times$ largeur $\times 0,75$ pour chacune des feuilles) ont été effectuées à Lusignan, en 1983, $15 \mathrm{j}$ avant la floraison, à floraison, 1 et 2 mois après floraison, en ne prenant en compte à chaque notation pour chacune des feuilles que la fraction non sénescente, verte, de celles-ci. La sénescence du $1^{\text {er }}$ entrenœud allongé de la base de la tige a été notée, en 1983, à Lusignan 1 et 2 semaines, 1, 1,5 et 2 mois après la floraison selon une échelle de 1 à 9 :

$1=$ entre 0 et 25 p. 100 de tissus sénescents.

$2=$ autour de 25 p. 100 .

$3=$ entre 25 et 50 p. 100 .

$4=$ autour de 50 p. 100 .

$5=$ entre 50 et 75 p. 100.

$6=$ autour de 75 p. 100

$7=$ entre 75 et 100 p. 100.

8 = autour de 100 p. 100.

9 = sénescence ayant gagné les nœuds adjacents.

Les notations de pourritures de tiges sont exprimées en pourcentages de plantes pourries par rapport au nombre total de plantes de la ligne. Les corrélations calculées sont les corrélations phénotypiques inter qui tiennent compte du modèle de l'analyse de variance.

\section{RÉSULTATS}

\section{A. Notations et pourriture des tiges}

Les 2 milieux retenus, Mons et St-Martin-de-Hinx, sont régulièrement favorables à un bon développement de la pourriture des tiges, le $1^{\text {er }}$ en raison de la sensibilisation des plantes par les basses températures d'arrière-saison et de la richesse du sol en Fusarium roseum culmorum, le $2^{\mathrm{e}}$ parce qu'il permet une bonne maturation des plantes dans un milieu riche en champignons parasites ou semi-parasites (ROUHANI et al., 1979, BARRIERE et al., 1981). Pour des raisons encore non connues, l'expression du symptôme pourriture de tiges ne se fait pas de façon aussi claire dans le milieu Lusignan.
Les notations de St-Martin-de-Hinx montrent une apparition plus précoce de la pourriture sur 2 génotypes, " Primeur » et " F16 $\times$ F19 » qui présentent 36 et $20 \mathrm{p}$. 100 de plantes attaquées $60 \mathrm{j}$ après la floraison, un peu plus précoce sur "Leader" avec 40 p. 100 d'attaque $75 \mathrm{j}$ après floraison, "Primeur " et «F16 × F19 » se situant alors à 68 p. 100 . Pour les autres génotypes l'attaque ne devient notable que $90 \mathrm{j}$ après floraison (tabl. 1). Les notations entre les 2 lieux sont bien corrélées $\left(r=0,86^{* *}\right)$, la plus faible notation à Mons de l'hybride "Monarque", génotype le plus tardif, s'expliquant sans doute par son niveau de maturité insuffisant, celle de "Primeur » étant plus difficile à expliquer. Les résultats sur les 2 lieux montrent la sensibilité élevée des hybrides " Primeur », « Leader ", « F16 × F19 », le comportement moyen de «Bruex » et de "Monarque», un bon ou assez bon niveau de résistance pour « Brulouis », « Dea » ou " Brussol ». Ils confirment le comportement agronomique habituel de ces variétés, si ce n'est pour "Bruex" jusqu'alors considéré comme résistant ou assez résistant.

TABLEAU 1

Pourcentage de plantes à tiges pourries 3 mois après floraison à St-Martin-de-Hinx (SMH) et Estrées-Mons (1983).

Percent of stalk-rotted plants 3 months after mid silking at St-Martin-de-Hinx (SMH, Southern France) and Estrées-Mons (Mons, Northern France).

\begin{tabular}{lcc}
\hline \hline & SMH & Mons \\
\hline Primeur & 86,7 & 44,0 \\
Leader & 78,4 & 77,3 \\
Brulouis & 14,3 & 12,0 \\
Brussol & 0,0 & 0,0 \\
Bruex & 46,7 & 42,7 \\
Dea & 6,7 & 9,3 \\
F16 $\times$ F19 & 89,3 & 65,3 \\
Monarque & 55,5 & 17,3 \\
F genotypes & $44,32^{* *}$ & $16,47^{* *}$ \\
\hline
\end{tabular}

\section{B. Rythme d'apparition des feuilles aux stades jeunes}

Ce critère de vitesse de mise en place de l'appareil photosynthétique pourrait être en relation avec la quantité totale de photosynthétats produits, dans la période préflorale en particulier, et donc être lié aux équilibres de transferts qui s'établissent dans la plante entière après la floraison. Les nombres de feuilles adultes (ligule visible), ou visibles, sont peu différents d'un génotype à l'autre dans les tout premiers stades, mais des écarts s'établissent progressivement et atteignent ainsi 0,6 et 1,4 feuilles $57 \mathrm{j}$ après le semis, et cela sans lien direct avec la précocité (tabl. 2). Si ce

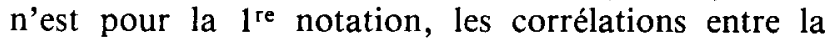
pourriture des tiges et les nombres de feuilles sont plus élevées pour les feuilles adultes que pour les feuilles visibles, en particulier pour les 2 dernières notations avec respectivement $\mathrm{r}=-0,76^{* *}$ et $-0,70^{* *}$ pour les feuilles adultes et $r=-0,39$ et $-0,30 \mathrm{NS}$ pour les feuilles visibles. La vitesse d'apparition des 
TABLEAU 2

Etude du rythme d'apparition des feuilles au stade jeune, entre 195 et 584 degrés-jours après le semis (base 6). Study of leaf appearance rate during early stages, from 195 to 584 degree-days over $6^{\circ}$ after sowing date.

A, V Nombre de feuilles adultes (A) ou visibles (V) pour une somme de température de... degrés-jour après le semis (base 6). Number of adults $(A)$ or appeared $(V)$ leaves for... degree-days over $6^{\circ}$ after sowing.

PEA, PEV Pente de la droite de régression du nombre de feuilles adultes et visibles sur la somme de température en base 6.

Regression slope of adults and appeared leaves on degree-days over $6^{\circ}$.

NF Nombre de feuilles présentes à floraison.

Number of leaves at mid-silking.

STF Somme de température en base 6 pour la période semis floraison.

Degree-days over $6^{\circ}$ for sowing mid-silking period.

\begin{tabular}{|c|c|c|c|c|c|c|c|c|c|c|c|c|c|c|c|c|}
\hline & \multicolumn{16}{|c|}{ Degrés-jours après le semis } \\
\hline & \multicolumn{2}{|c|}{195} & \multicolumn{2}{|c|}{287} & \multicolumn{2}{|c|}{387} & \multicolumn{2}{|c|}{436} & \multicolumn{2}{|c|}{509} & \multicolumn{2}{|c|}{584} & \multirow[t]{2}{*}{ PEA } & \multirow[t]{2}{*}{ PEV } & \multirow[t]{2}{*}{ NF } & \multirow[t]{2}{*}{ STF } \\
\hline & A & $\mathrm{v}$ & A & $\mathrm{v}$ & A & $\mathrm{v}$ & A & $\mathrm{v}$ & A & $\mathrm{v}$ & A & V & & & & \\
\hline Primeur & 2,0 & 5,0 & 3,5 & 6,9 & 4,7 & 8,7 & 5,2 & 9,9 & 6,1 & 11,2 & 7,3 & 12,1 & 1,31 & 1,87 & 12,5 & 789 \\
\hline Leader & 2,0 & 4,8 & 3,0 & 6,1 & 4,3 & 8,0 & 5,0 & 9,5 & 6,0 & 11,0 & 7,0 & 12,2 & 1,30 & 2,00 & 13,0 & 841 \\
\hline Brulouis & 2,3 & 5,0 & 4,0 & 6,8 & 5,0 & 8,9 & 5,9 & 10,1 & 6,8 & 11,9 & 7,9 & 13,0 & 1,40 & 2,10 & 14,6 & 805 \\
\hline Brussol & 2,1 & 5,0 & 3,9 & 6,8 & 5,0 & 8,7 & 5,9 & 9,8 & 6,8 & 11,4 & 8,1 & 13,1 & 1,47 & 2,07 & 14,6 & 832 \\
\hline Bruex & 2,0 & 4,8 & 3,2 & 6,3 & 4,7 & 8,3 & 5,6 & 9.7 & 6,5 & 11,4 & 7,4 & 12,5 & 1,41 & 2,08 & 14,0 & 841 \\
\hline Dea & 2,0 & 4,9 & 3,6 & 6,7 & 5,0 & 8,7 & 5,7 & 9,9 & 6,8 & 11,6 & 7,9 & 13,4 & 1,49 & 2,16 & 15,0 & 832 \\
\hline Monarque & 2,0 & 4,9 & 3,0 & 6,0 & 4,9 & 8,2 & 5,4 & 9,0 & 6,2 & 10,5 & 7,0 & 12,0 & 1,32 & 1,87 & 17,0 & 921 \\
\hline F génotypes & $3,10^{*}$ & $6,82^{* *}$ & $20,68^{* *}$ & $13,34^{* *}$ & $4,21^{*}$ & 2,09 & $16,63^{* *}$ & $3,33^{*}$ & $6,68^{* *}$ & $3,70^{*}$ & $7,89 * *$ & $9,30^{* *}$ & $5,55^{* *}$ & $9,79^{* *}$ & $103,34^{* *}$ & - \\
\hline
\end{tabular}

feuilles, estimée par la pente de la droite de régression du nombre de feuilles sur la somme de températures, n'est pas liée à la précocité. Mais, de même, il y a une tendance à la liaison entre la vitesse d'apparition des feuilles adultes et la résistance $(r=-0,55$ NS entre pente et pourriture), liaison qui n'est pas mise en évidence pour la vitesse d'apparition des feuilles visibles $(r=-0,09$ NS).

\section{Evolution des surfaces foliaires}

Trois phases peuvent en principe être distinguées dans l'évolution de l'indice de surface foliaire (LAI = surface de feuilles par unité de surface cultivée), d'une culture de maïs (EIK \& HANWAY, 1965 ; DALE et al., 1980 ; RUGET, 1981 ; BLANCHET et al., 1982). La $1^{\text {re }}$ phase, exponentielle, correspond à la mise en place du système foliaire entre le semis et la floraison. La $2^{\mathrm{e}}$, dont la durée est variable selon le milieu et les génotypes, correspond à une décroissance assez lente de 15 à $20 \mathrm{p} .100 \mathrm{du} \mathrm{LAI}$. La $3^{\mathrm{e}}$ est une phase de croissance plus rapide et linéaire du LAI, dont l'intensité et la précocité varie avec les conditions de milieu (TOLLENAAR, 1977).
Les différences de LAI entre les génotypes observés sont significatives à $1 \mathrm{p}$. 100, sauf pour la notation faite 1 mois après la floraison. Les valeurs de LAI obtenues à floraison sont classiques pour les hybrides et le milieu considéré, les valeurs habituelles se situant autour de 2,5 à 3,0 (DAYNARD \& TOLLENAAR, 1983), avec une valeur élevée pour "Monarque » qui est peut-être due à son caractère semi-prolifique (PHILIPPE, 1980 ; BARRIERE et al., 1984 (tabl. 3, fig. 1).

\section{TABLEAU 3}

Indices de surface foliaire 15 jours avant floraison, à floraison et 2 mois après floraison.

Leaf area index 15 days before midsilking, at midsilking, 2 months after midsilking.

\begin{tabular}{lccc}
\hline & -15 jours & floraison & +2 mois \\
Primeur & 1,29 & 2,70 & 0,72 \\
Leader & 1,07 & 2,84 & 1,24 \\
Brulouis & 1,06 & 2,80 & 1,50 \\
Brussol & 1,07 & 2,92 & 2,05 \\
Bruex & 1,29 & 3,26 & $1,68-$ \\
Dea & 1,10 & 3,12 & 2,10 \\
F16 $\times$ F19 & 1,31 & 3,13 & 1,11 \\
Monarque & 1,09 & 3,77 & 2,25 \\
F génotypes & $5,43^{* *}$ & $5,17^{* *}$ & $5,00^{* *}$ \\
\hline \hline
\end{tabular}

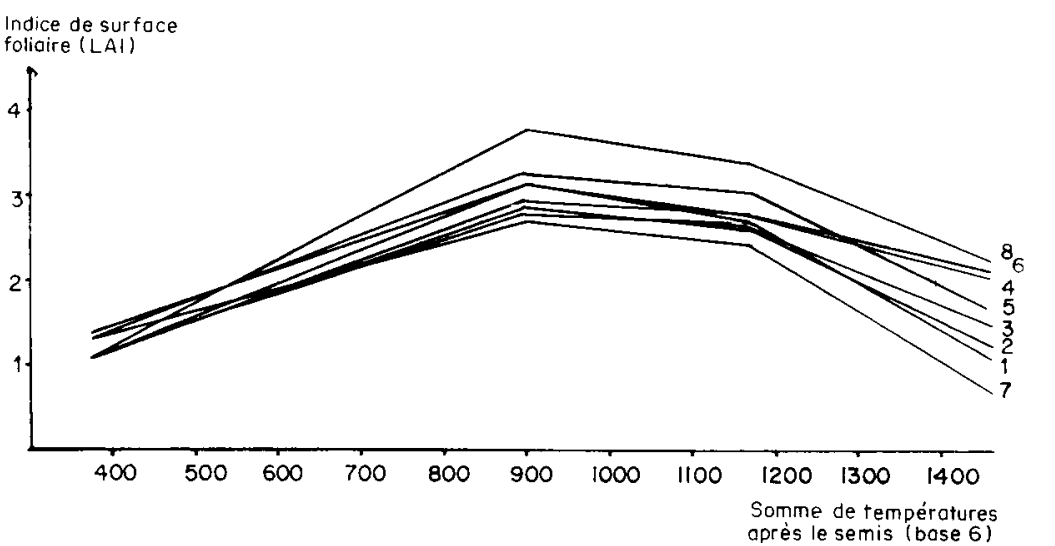

Figure 1

Evolution des indices de surface foliaire.

Change in leaf area index.

$I=$ Primeur $; \quad 5=$ Bruex .

$2=$ Leader $; \quad 6=$ Dea .

$3=$ Brulouis ; $\quad 7=$ Fl6 $\times F 19$.

$4=$ Brussol $; \quad 8=$ Monarque.

Même décodage des génotypes pour les figures 1, 2 , 3 et 4. 
La corrélation entre le LAI $15 \mathrm{j}$ avant la floraison et la note moyenne de pourriture des tiges est faible et positive ( $r=0,49 \mathrm{NS})$, celle entre le LAI à floraison et la pourriture des tiges est pratiquement nulle $(r=-0,09 \mathrm{NS}$ ), alors que celle entre le LAI 2 mois après la floraison et la pourriture des tiges est assez élevée et négative $\left(\mathrm{r}=-0,73^{* *}\right)$. Ces résultats montrent en particulier l'existence, dans cet échantillon de variétés, d'une liaison génétique entre le LAI 2 mois après la floraison, qui mesure l'aptitude à maintenir un appareil photosynthétique fonctionnel en fin de végétation, et la résistance à la pourriture des tiges. Une diminution du LAI de 3 à 2 correspond à une diminution de l'interception du rayonnement incident visible de 88 à 75 p. 100 (BONHOMME et al., 1982 ; DAYNARD \& TOLLENAAR, 1983), rayonnement qui est lui-même nettement décroissant en fin de végétation (VARLET-GRANCHER, 1982 ; TOLlENAAR, 1983). La liaison positive entre le LAI avant floraison et la pourriture des tiges est due aux valeurs plus élevées des 3 hybrides sensibles ou assez sensibles "F16 × F19 », " Primeur » et « Bruex » à ce stade. Ceci est apparemment contradictoire avec les observations faites sur les vitesses d'apparition de feuilles, et tout se passe comme si ces 3 génotypes avaient, à partir d'un stade situé peu avant floraison, une précocité de maturation physiologique plus élevée. Enfin, il y a une corrélation entre le LAI en fin de végétation et la productivité finale en plante entière $\left(r=0,68^{*}\right)$, mais aussi entre la LAI à floraison et la productivité finale en plante entière $\left(r=0,65^{*}\right)$. Ces liaisons ne peuvent pas être expliquées seulement par un effet de précocité, les productivités plante entière finales étant par ailleurs bien corrélées entre l'essai de Lusignan et celui d'Estrées-Mons $\left(r=0,74^{* *}\right)$.

\section{Evolution de la sénescence des tiges}

Les travaux réalisés sur des hybrides de lignées nord-américaines tardives ont montré que l'apparition de zones sénescentes, caractérisées par un faible nom- bre de cellules vivantes, était précoce et se produisait différemment chez les génotypes sensibles ou résistants aux pourritures des tiges. Les génotypes résistants présentaient ainsi une sénescence de la base des tiges plus tardive, plus faible, dont l'extension était plus lente (PAPPELIS \& SMITH, 1963 ; PAPPELIS, 1965 ; WYSONG \& HOOKER, 1966). Les teneurs en eau des tiges sont aussi un indicateur de sénescence et ont été reliées à la pourriture des tiges (PAPPELIS \& SMITH, 1963).

Dans notre échantillon de génotypes, la sénescence des tiges débute effectivement précocement puisque, une semaine après la floraison, le minimum de sénescence de l'entrenœud de la base des tiges est de un peu plus de 25 p. 100 (« Brussol ») et le maximum se situe autour de 75 p. 100 («F16 × F19») (tabl. 4, fig. 2). Les 3 génotypes les plus sensibles (« Primeur», "Leader », " F16 × F19 ») ont, d'une manière générale, le niveau de sénescence le plus élevé. Le génotype assez sensible «Bruex » se comporte dans la $1^{\text {re }}$ partie

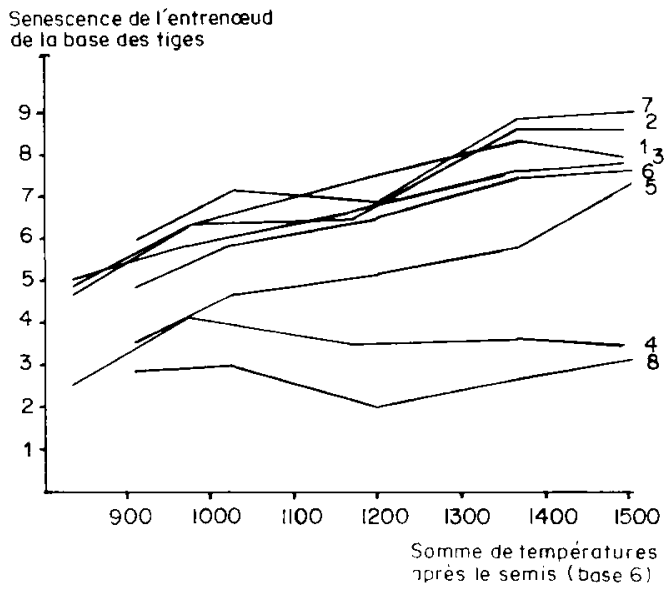

Figure 2

Evolution de la sénescence du ler entrenceud allongé de la base des tiges.

Progress of senescence of first basal elongate internode of the stalk.

TABLEAU 4

Sénescence du $I^{\text {er }}$ entrenœud allongé de la base de la tige (notes de 1 à 9) et teneur en matière sèche des tiges après la floraison. Senescence of first elongate internode of basal stalk (scores from I to 9) and stalk percent of dry matter after mid-silking.

\begin{tabular}{|c|c|c|c|c|c|c|c|c|}
\hline & \multicolumn{5}{|c|}{$\begin{array}{l}\text { Sénescence de l'entrencud de la base } \\
\text { Basal internode senescence }\end{array}$} & \multicolumn{3}{|c|}{$\begin{array}{l}\text { Teneur en matière sèche des tiges } \\
\text { Stalk percent of dry-matter }\end{array}$} \\
\hline & 7 jours & 15 jours & 30 jours & 45 jours & 60 jours & 1 mois & 2 mois & 3 mois \\
\hline Primeur & 4,8 & 6,3 & 7,3 & 8,3 & 8,0 & 16,5 & 24,1 & 40,9 \\
\hline Leader & 4,7 & 6,3 & 6,5 & 8,7 & 8,7 & 17,5 & 25,5 & 41,8 \\
\hline Brulouis & 5,0 & 5,8 & 6,7 & 7,7 & 7,8 & 19,6 & 22,3 & 37,0 \\
\hline Brussol & 2,5 & 4,2 & 3,5 & 3,7 & 3,5 & 20,0 & 22,4 & 35,8 \\
\hline Bruex & 3,5 & 4,7 & 5,2 & 5,8 & 7,3 & 17,1 & 20,5 & 34,7 \\
\hline Dea & 4,8 & 5,8 & 6,5 & 7,5 & 7,7 & 19,6 & 21,1 & 35,0 \\
\hline $\mathrm{F} 16 \times \mathrm{F} 19$ & 6,0 & 7,2 & 6,8 & 8,8 & 9,0 & 17,8 & 26,9 & 48,4 \\
\hline Monarque & 2,8 & 3,0 & 2,0 & 2,7 & 3,2 & 19,5 & 18,7 & 31,9 \\
\hline$F$ génotypes & $28,26 * *$ & $25,93^{* *}$ & $34,22 * *$ & $53,04 * *$ & $58,39 * *$ & $6,09^{* *}$ & $7,67^{* *}$ & $8,30^{* *}$ \\
\hline
\end{tabular}


de la période post-florale comme un type peu sénescent, puis évolue assez rapidement vers des niveaux de sénescence assez élevés. "Monarque ", également assez sensible, conserve toujours un état de nonsénescence élevé, en raison en partie de sa plus grande tardiveté qui aurait sans doute nécessité une notation supplémentaire. Enfin, ces courbes montrent que 2 évolutions distinctes de sénescence des tiges peuvent permettre l'expression de la résistance puisque «Brulouis » et «Dea » évoluent comme les génotypes sensibles, à un niveau un peu moindre, et que «Brussol " conserve un niveau de non-sénescence élevé jusqu'à la récolte. Les corrélations entre chacune des notations et la pourriture des tiges sont comprises entre 0,39 et 0,55 , les valeurs les plus élevées étant obtenues pour les 2 dernières notations.

Les 3 génotypes sensibles étudiés ont un dessèchement de la tige plus précoce et plus intense que les autres génotypes, l'hybride «F16 × F19 » ayant en particulier une intensité élevée de dessèchement (fig. 3). En revanche, les évolutions des teneurs en matière sèche des tiges ne distinguent pas les 2 génotypes assez sensibles des génotypes résistants, mais ceci peut, en partie, être dû à des effets de tardiveté, en particulier pour "Monarque ". La teneur en matière sèche des tiges des 2 derniers prélèvements ( 2 et 3 mois après floraison) est corrélée positivement avec la pourriture des tiges $\left(r=0,60^{*}\right.$ et $\left.0,71^{*}\right)$, avec la sénescence 1,5 mois après floraison $\left(r=0,74^{* *}\right.$ et $0,75^{* *}$ ) et avec la sénescence 2 mois après floraison ( $r=0,64^{*}$ et $\left.0,66^{*}\right)$. Il y a, au contraire, une corrélation négative élevée entre la teneur en matière sèche des' tiges 1 mois après la floraison et la pourriture des tiges $\left(r=-0,77^{* *}\right)$, les variétés « Primeur ", " Leader ", «Bruex » et «F16 × F19 » ayant en particulier des teneurs inférieures à la moyenne du prélèvement à ce stade. A Estrées-Mons, la corrélation entre teneur en matière sèche des tiges 3 mois après floraison et pourriture de tiges est également élevée $\left(r=0,80^{* *}\right)$, de même que celle entre les teneurs 3 mois après floraison à Lusignan et Estrées-Mons $\left(r=0,75^{* *}\right)$. En revanche, la corrélation entre teneur en matière sèche 1 mois après floraison et pourriture

Teneur en matière sèche

des tiges (p. 100 )

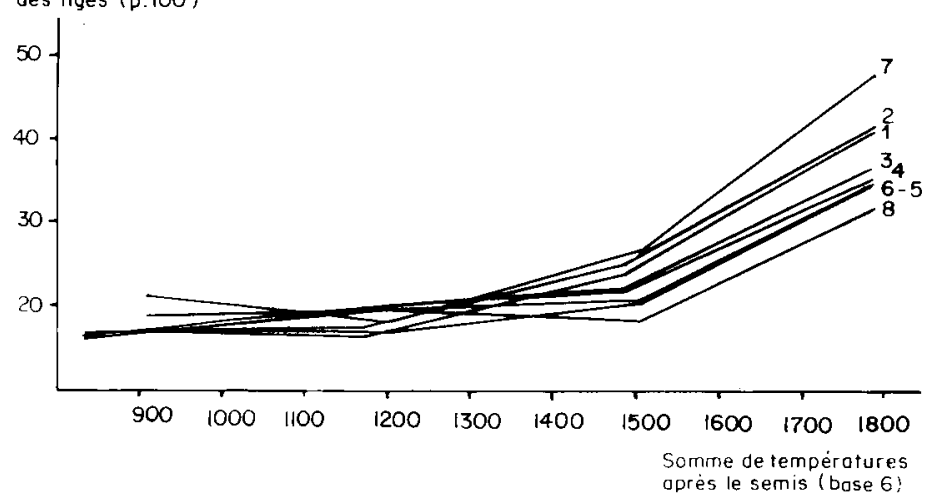

Figure 3

Evolution de la teneur en matière sèche des tiges.

$\%$ dry matter of stalk. des tiges n'y est pas significative $(r=0,22)$, la période d'1 mois après floraison à Estrées-Mons n'étant toutefois pas directement comparable avec celle d' 1 mois après floraison à Lusignan.

\section{E. Evolution des rapports épi/plante entière}

Un rapport épi/plante entière plus élevé dès 30 à $45 \mathrm{j}$ après la floraison, indiquant un déséquilibre nutritionnel plus important et plus précoce, semble être une des caractéristiques des génotypes sensibles aux pourritures des tiges (WALL \& MORTIMORE, 1965 ; GATES, 1970 ; BARRIERE et al., 1981).

Les rapports épi/plante entière 1 mois après la floraison ne permettent pas de distinguer parmi les génotypes étudiés, les types sensibles des types résistants. En revanche 2 mois après la floraison, les 3 génotypes sensibles présentent les rapports les plus élevés, et 3 mois après la floraison ces hybrides se distinguent nettement des autres avec des rapports épi/plante entière supérieurs à 0,7 , les hybrides «Brussol » et «Brulouis » ayant les rapports les plus faibles, «Dea ", «Bruex » et «Monarque » étant très proches (tabl. 5, fig. 4). La corrélation entre rap-

TABLEAU 5

Evolution des rapports épi/plante entière après la floraison femelle. Whole-ear/whole-plant ratio 1,2 and 3 months after midsilking.

\begin{tabular}{llll}
\hline & 1 mois & 2 mois & 3 mois \\
Primeur & 0,60 & 0,68 & 0,73 \\
Leader & 0,57 & 0,68 & 0,74 \\
Brulouis & 0,53 & 0,65 & 0,66 \\
Brussol & 0,47 & 0,60 & 0,59 \\
Bruex & 0,50 & 0,65 & 0,67 \\
Dea & 0,51 & 0,67 & 0,68 \\
F16 $\times$ F19 & 0,52 & 0,67 & 0,73 \\
Monarque & 0,44 & 0,62 & 0,68 \\
F génotypes & $5,09 * *$ & $6,29 * *$ & $17,00^{* *}$ \\
\hline \hline
\end{tabular}

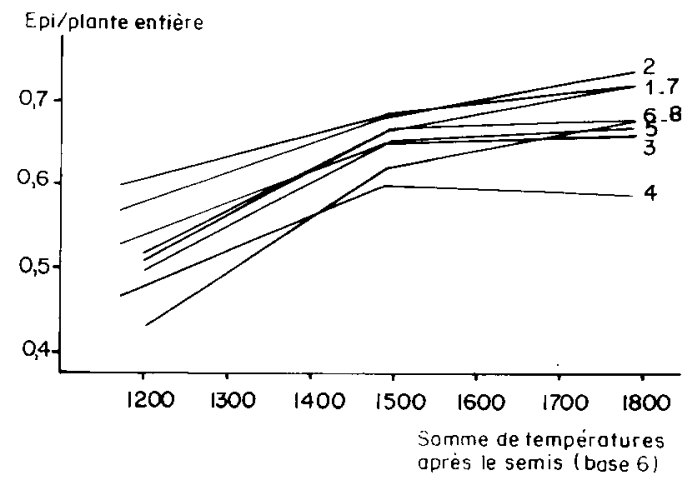

Figure 4

Evolution des rapports épi/plante entière.

Whole ear/whole plant ratio 
port épi/plante entière 3 mois après la floraison et pourriture des tiges est élevée $\left(r=0,87^{* *}\right)$, de même que celle, négative, avec le LAI au même stade $\left(\mathrm{r}=-0,68^{*}\right)$ ou celle avec la sénescence 2 mois après la floraison $\left(r=0,69^{*}\right)$. L'évolution de ces rapports au cours des cycles de végétation peut être très différente d'un hybride à l'autre. Ainsi, le rapport épi/plante entière de «Brussol » n'augmente que de 0,11 point entre 1 et 3 mois après la floraison, celui de «Brulouis » augmente de 0,13, tandis que celui de «F16 × F19», qui a une productivité plante entière finale proche de celle de «Brulouis», augmente de 0,20 . Enfin, il ne semble pas y avoir de liaison entre la productivité grain et le rapport épi/plante entière ( $\mathrm{r}=-0,15 \mathrm{NS}$ ), mais une tendance à la liaison négative avec le rendement plante entière ( $r=-0,46 \mathrm{NS})$. De plus, il n'y a pas de liaison entre productivité grain ou plante entière et pourriture des tiges $(r=0,14$ et - 0,20 NS).

\section{DISCUSSION}

Une analyse en composantes principales réalisée à partir de la matrice des corrélations phénotypiques classiques entre 16 variables (fig. 5) donne une répartition graphique des génotypes qui schématise assez clairement l'ensemble des observations. L'axe 1, qui explique 65 p. 100 de la variation, est très corrélé avec les différentes notations de sénescence ( $r$ compris entre 0,75 et 0,96 ), avec les teneurs en matière sèche des tiges 2 et 3 mois après floraison $(r=0,83$ ), avec les rapports épi/plante entière 2 et 3 mois après floraison $(r=0,89$ et 0,76$)$, avec la pourriture des tiges $(\mathrm{r}=0,70)$ et négativement avec le LAI à 3 mois $(r=-0,92)$. L'axe $2(16,1$ p. 100) est corrélé avec la productivité grain et plante entière $(\mathrm{r}=0,96$ et 0,82$)$. L'axe 3 (9,8 p. 100) est corrélé avec la teneur en matière sèche des tiges 1 mois après la floraison $(r=0,65)$ et négativement avec la pourriture des tiges $(\mathrm{r}=-0,60)$. Sur les 2 graphes (axe $1 \times$ axe 2 et axe $1 \times$ axe 3 ), les variétés sensibles se situent sur la partie droite, plus ou moins proches en fonction de leurs différences de productivité, les variétés résistantes à tiges sénescentes se situent au centre, proches de « Bruex » qui a la même physiologie de tiges, tandis que «Monarque » et «Brussol » dont les tiges demeurent non sénescentes sont à la gauche des graphiques. Le graphe axe $1 \times$ axe 3 distingue particulièrement bien à la fois les différents types physiologiques et les différentes classes de sensibilité.

La recherche par une méthode de régression progressive des variables les plus explicatives de la pourriture des tiges parmi les 16 précédentes montre que les 3 premières variables retenues sont dans l'ordre le rapport épi/plante entière 3 mois après floraison, la teneur en matière sèche des tiges 3 mois après la floraison et la teneur en matière sèche des tiges 1 mois après la floraison avec des coefficients de corrélations multiples valant successivement $0,83,0,88$ et 0,92 . La sénescence 1,5 mois après floraison et la teneur en matière sèche des tiges 2 mois après floraison sont ensuite retenues.
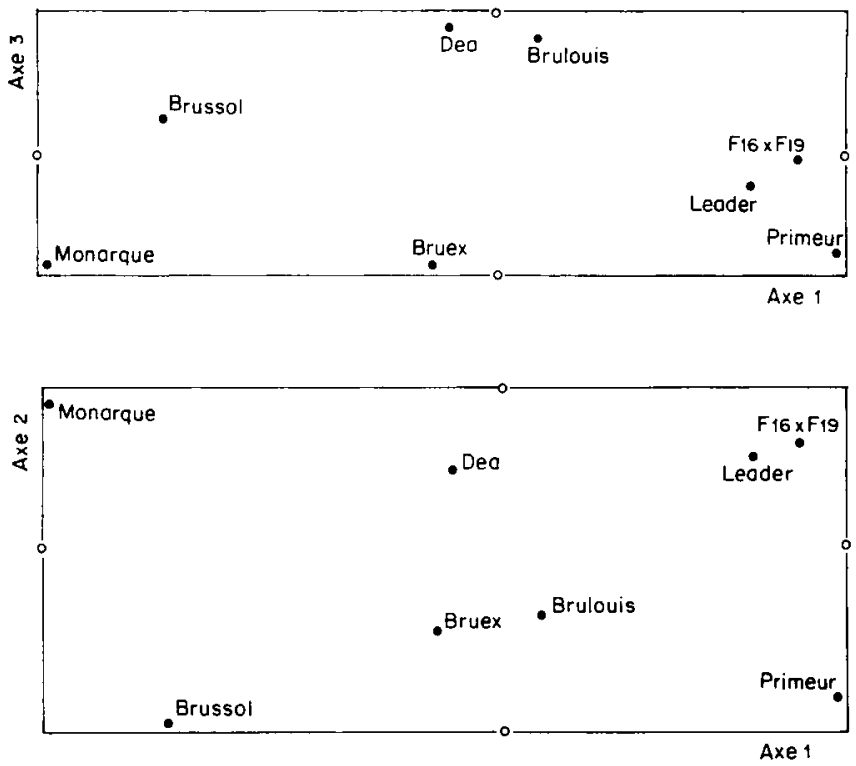

Figure 5

Analyse en composantes principales avec 10 variables explicatives. Principal components analysis with 16 significant variables.

Liste des variables

- Teneur en matière sèche des tiges 1, 2 et 3 mois après floraison. - Rapport épilplante entière $1,2,3$ mois après floraison.

- Sénescence de l'entrenœud de la base des tiges 7, 15, 30, 45 et $60 j$ après floraison.

- Indice de surface foliaire à floraison et 2 mois après floraison. - Rendement plante entière et rendement grain 3 mois après floraison.

- Pourcentage moyen de tiges pourries à la récolte.

List of variables

- Stalk percent of dry-matter 1, 2 and 3 months after mid-silking. - Whole ear/whole plant ratio $I, 2$ and 3 months after midsilking.

- Senescence of first elongate basal internode 7, 15, 30, 45 and 60 days after mid-silking.

- Leaf area index at mid-silking and 2 months after mid-silking.

- Whole plant and grain yield 3 months after midsilking.

- Average percentage of rotted stalks 3 months after midsilking.

Une disponibilité réduite en sucres ou en ATP peut exercer un effet initiateur direct de la sénescence des organes carencés comme la compétition entre organes pour les nutriments peut induire la sénescence des organes les plus âgés (LEVITT, 1972; THOMAS \& STODDART, 1980). L'arrêt de croissance des racines du maïs après la floraison est ainsi considéré comme la conséquence de la forte demande en nutriments de la partie aérienne et sera suivi de leur sénescence (FoTH, 1962 ; AUNG, 1974), parallèlement d'ailleurs à une forte multiplication de la mycoflore racinaire (HORNBY \& UlLSTRUP, 1967). La sénescence des tiges peut, de même, être envisagée comme la conséquence de déséquilibres nutritionnels qui résultent d'une inadéquation entre la demande de l'épi et l'offre par la photosynthèse ; ceci rejoint tout à fait l'hypothèse de DODD (1980) sur les liaisons entre stress photosynthétique, équilibre des transferts et pourriture des tiges. Ce déséquilibre nutritionnel pouvant avoir lieu soit en raison d'un déclin trop précoce de la photosynthèse après diminution du LAI ou de la photosynthèse ellemême, soit, à photosynthèse sensiblement constante, en raison d'une demande trop importante de l'épi. Les 
fortes intensités ou les niveaux élevés de transferts et la diminution plus rapide du LAI chez les génotypes sensibles montrent que ces facteurs peuvent agir simultanément et entraîner la sénescence des organes carencés.

La sénescence des tiges est une évolution normale de la vie de la plante, mais l'existence de corrélations moyennes (voisines de 0,55 ) entre la sénescence de l'entre-nœud de la base des tiges 1,5 et 2 mois après floraison et la pourriture des tiges 3 mois après floraison laisse penser que c'est son apparition trop précoce qui indique un désordre métabolique et non son existence en soit; d'ailleurs, des génotypes résistants comme «Dea » et " Brulouis » présentent un niveau final de sénescence des tiges assez élevé et, en régression progressive, c'est la note de sénescence 1,5 mois après floraison (et non 2 mois) qui est introduite parmi les premières variables explicatives, après le rapport épi/plante entière.

Les teneurs en matière sèche des tiges 2 et 3 mois après la floraison sont aussi des variables explicatives de la pourriture des tiges, corrélées positivement avec celle-ci. Des teneurs faibles en eau des tiges indiquent une perte de turgescence des tissus et sont donc une autre approche et un autre indicateur de la sénescence des plantes. La teneur en matière sèche des tiges 1 mois après floraison est également une variable explicative, mais corrélée négativement. Ce qui veut dire que ce sont les faibles teneurs en matière sèche qui sont, à ce stade, liées à la pourriture des tiges. Ces faibles teneurs peuvent correspondre à des prélèvements plus importants et plus précoces des réserves de la tige au profit du jeune épi chez les génotypes sensibles et donc à l'apparition plus précoce d'un déséquilibre nutritionnel initiateur de sénescence.

Pour des génotypes comme «F16 × F19 », connus pour leur flétrissement élevé en fin de végétation, il semble que l'on puisse considérer que c'est une physiologie particulière, avec un début de sénescence précoce et des transferts précoces et intenses vers l'épi, qui conduit à une sénescence prématurée de la plante entière, suivie de son dessèchement et de son flétrissement. Le niveau élevé de pourriture des tiges observé sur ce génotype en fin de végétation semble donc à relier d'abord à ce type de fonctionnement physiologique, puis à une sensibilité sans doute élevée des tissus sénescents ou en voie de sénescence à des parasites parmi lesquels figure peut être $F$. moniliforme. Il paraîtrait alors possible d'utiliser une physiologie de ce type pour l'amélioration des maïs-fourrage, cet hybride ayant en particulier un niveau de productivité en plante entière égal aux témoins avec, en raison de sa sénescence précoce, une teneur en matière sèche améliorée de 4 à 6 points (GALLAIS, 1983) et cela sans présenter de pourriture des tiges notable à la date de récolte des maïs ensilage. L'utilisation de tels géniteurs, en combinaison avec des sources de moindre sensibilité intrinsèque aux parasites, permettrait d'utiliser le potentiel très important de productivité du matériel tardif tout en maintenant une précocité suffisante des hybrides commerciaux, en triant simultanément sur la valeur alimentaire (digestibilité in vitro par exemple).

\section{CONCLUSION}

Bien que ces essais aient été réalisés sur un nombre limité de génotypes, ils semblent bien confirmer que ce sont des facteurs physiologiques intervenant tout au long de la vie de la plante qui conditionnent la sensibilité ou la résistance du maïs aux pourritures des tiges. Mais il ne semble pas exister simplement une physiologie de plante sensible et une physiologie de plante résistante. Ce sont les équilibres ou les déséquilibres qui s'instaurent globalement dans la plante entière au cours du cycle sous l'effet de chacun de ces facteurs qui conduisent à la résistance ou à la sensibilité. Toutefois, pour des niveaux de déséquilibres physiologiques internes égaux, il peut exister des résistances différentes aux facteurs externes de désordre métabolique comme la sécheresse ou les basses températures (sans gel) qui conduisent, dans certains cas, à des arrêts irréversibles de la photosynthèse. De même, il existe, de façon presque certaine, des niveaux différents de résistance plus ou moins passive des tissus en cours de sénescence aux champignons semi-parasites impliqués dans la pourriture des tiges, à niveau de sénescence égal.

La sélection de génotypes résistants, parmi des effectifs importants, ne semble donc pouvoir se faire que par des notations tardives de pourriture sur des génotypes hétérotiques dans un milieu favorable à l'expression des symptômes. En revanche, la compréhension au niveau physiologique de la résistance à l'état hybride d'une lignée nécessitera des notations au cours de la période postflorale, dont les deux plus simples à réaliser, avec un bon niveau explicatif, sont sans doute les mesures d'évolution de la teneur en matière sèche des tiges et du LAI.

Reçu le 2 août 1984 Accepté le 26 novembre 1984.

\section{RÉFÉRENCES BIBLIOGRAPHIQUES}

Aung L. H., 1974. Root-shoot relationships, 29-61. In E. W. Garson : "The plant root and its environment ». University of Virginia.

Barrière Y., Panouille A., Cassini R., 1981. Relations source-puits et sélection du maïs pour la résistance à la pourriture des tiges. Agronomie, 1 (8), 707-711.

Barrière Y., Vincourt D., Montalant Y., 1984. Etude de la prolificité en épis du mais dans le premier cycle de sélection de deux populations précoces. Agronomie, 4 (4), 355-360.
Blanchet R., Merrien A., Gelfi N., Cavalie G., Courtiade B., Puech J., 1982. Estimation et évolution comparée de l'assimilation nette des couverts de maïs (Zea mays L.), tournesol (Helianthus annuus L.) et soja (Glycine max (L.) Merrill au cours de leurs cycles de développement. Agronomie, 2 (2), 149-154.

Bonhomme R., Ruget F., Berieux M., Vincourt P., 1982. Relations entre production de matière sèche aérienne et énergie interceptée chez différents génotypes de maïs. C. R. Acad. Sci. Paris, t. 294, série 3, 393-398. 
Dale R. F., Coelho D. T., Gallo K.P., 1981. Prediction of daily green leaf area index for corn. Agron. J., 72, 999-1005.

Daynard T. B., Tollenaar M., 1983. Prospects for improving the productivity of early maturing maize. Colloque physiologie du maïs, Royan, 15, 16, 17 mars 1983

Dodd J. L., 1980. The role of plant stresses in development of corn stalk rots. Plant Dis., 64 (6), 533-537.

Eik K., Hanway J.J., 1965. Some factors affecting development and longevity of leaves of corn. Agron. J., 57, 7-12.

Foth H. D., 1962. Root and top-growth of corn. Agron. J., 54, 49 53.

Gallais A., 1983. Amélioration du maïs fourrage. Agromais, 20, $11-$ 12.

Gates, 1970. Relationships between pith cell condition as assessed by tetrazolium chloride and incidence of Gibberella stalk rot in corn. Can. J. Plant Sci. 50, 679-684.

Hornby D., Ullstrup A. J., 1967. Fungal populations associated with maize roots. Quantitative rhizosphere data for genotypes differing in root rot resistance. Phytopathology, 57, 76-82.

Levitt J., 1972. Chilling injury and resistance, 27-43. In Response of plant to environmental stresses. Academic Press New-York.

Pappelis A. J., 1965. Relationship of seasonal changes in pith conditions ratings and density to Gibberella stalk rot of corn. Phytopathology, 55, 623-626.

Pappelis A. J., Smith F. G., 1963. Relationship of water content and living cells to spread of Diplodia zeae in corn stalks. Phytopathology, 53, 1100-1105.
Philippe F., 1980. Contribution à l'étude de la prolificité chez le maïs. Mémoire DAA, ENSA Montpellier, I.N.R.A. Lusignan, 83 p.

Rouhani H., Davet P., Poinso B., Beyries A., Messiaen C. M., 1979. Inventaire et évaluation du pouvoir pathogène des composants de la microflore fongique sur racines de maïs en France. Ann. Phytopathol., 11 (1), 69-93.

Ruget F., 1981. Respiration de croissance et respiration d'entretien au cours d'un cycle de végétation chez le maïs. Thèse Doct. Ing., Univ. Paris VII, 86 p.

Thomas H., Stoddart J. L., 1980. Leaf senescence. Annu. Rev. Plant Physiol., 31, 83-111.

Toltenaar M., 1977. Sink-source relationships during reproductive development in maize. A review. Maydica, 22, 49-75.

Tollenaar M., 1983. Potential vegetative productivity in Canada. Can. J. Plant Sci., 63, 1-10.

Varlet-Grancher C., 1982. Analyse du rendement de la conversion de l'énergie solaire par un couvert végétal. Thèse $\mathrm{n}^{\circ} 2593$, Université de Paris-Sud, Orsay, 144 p.

Wall R. E., Mortimore C. G., 1965. The growth pattern of corn in relation to resistance to root and stalk rot. Can. J. Bot., 43, 1277 . 1283.

Wysong D. S., Hooker A. L., 1966. Relation of soluble solids content and pith condition to Diplodia stalk rot in corn hybrids. Phytopathology, 56, 26-35. 